\title{
Determination of Diffraction Loss over Isolated Doubled Edged Hill Using the ITU-R P.526-13 Method for Rounded Edge Diffraction
}

\author{
Ogungbemi Emmanuel Oluropo ${ }^{1}$, Ikechukwu H. Ezeh ${ }^{2}$, Chibuzo Promise Nkwocha ${ }^{3}$ \\ ${ }^{1}$ Department of Electrical/Electronic and Computer Engineering, University of Uyo, Uyo, Nigeria \\ ${ }^{2}$ Department of Electrical Engineering, Imo State University (IMSU), Owerri, Nigeria \\ ${ }^{3}$ Department of Chemical Engineering Federal University of Technology, Owerri (FUTO), Owerri, Nigeria
}

Email address:

promisechibuzor413@yahoo.com (C.P. Nkwocha)

\section{To cite this article:}

Ogungbemi Emmanuel Oluropo, Ikechukwu H. Ezeh, Chibuzo Promise Nkwocha. Determination of Diffraction Loss over Isolated Doubled Edged Hill Using the ITU-R P.526-13 Method for Rounded Edge Diffraction. American Journal of Software Engineering and Applications. Vol. 6, No. 2, 2017, pp. 56-60. doi: 10.11648/j.ajsea.20170602.18

Received: January 8, 2017; Accepted: January 18, 2017; Published: June 12, 2017

\begin{abstract}
In this paper, Recommendation ITU-R P.526-13 rounded edge diffraction loss method is used to determine the diffraction loss over a double edged hilltop in the path of $6 \mathrm{GHz}$ C-band microwave signal. The computation is based on the path profile with path length of $6188.665 \mathrm{~m}$. The path profile has maximum elevation of $412.75 \mathrm{~m}$ and it occurred at a distance of $2877.3 \mathrm{~m}$ from the transmitter. The line of sight clearance height is $35.393521 \mathrm{~m}$ and occultation distance is $532.203 \mathrm{~m}$. The diffraction loss computed for the double edged hilltop using the Recommendation ITU-R P.526-13 model is $42.563065 \mathrm{~dB}$.
\end{abstract}

Keywords: Diffraction Loss, Diffraction Parameter, Doubled Edged Hill, ITU-R P.526-13 Method, Rounded Edge Diffraction

\section{Introduction}

In wireless communication system, as signal propagates along the path from transmitter to the receiver, it experiences reduction in signal strength which is generally referred to as path loss [1-5]. The path loss may include propagation losses caused by the natural expansion of the radio wave front in free space, absorption losses, as well as diffraction losses when part of the radio wave front is obstructed by an opaque obstacle [6-12]. In other to estimate the diffraction loss caused by isolated obstacles like hills, mountains, buildings, such isolated obstacles are modeled as single knife edge obstructions [13-15]. However, in reality, the obstruction presents more diffraction loss than the single knife edge approximation. In that case, rounded edge diffraction loss approximation may be applied to such isolated obstacles.

Over the years, several methods for determination of rounded edge diffraction loss have been developed. One of the popular approaches is a method to determine the excess diffraction loss above the knife edge diffraction loss. The access diffraction loss can be computed according to Hacking method [1], [17]. Wait method is another method for computing the access diffraction loss in addition to the knife edge approximation [18], [19]. However, in this paper, the method presented by the International Telecommunication Union (ITU) for computing diffraction loss over single rounded obstacle is used to compute the diffraction loss over double edged hilltop [20].

In all the methods of computing diffraction loss over rounded edge, the basic approach is to fit a rounded edge to the vertex of the obstruction and then use the radius of curvature of the rounded edge to compute the diffraction loss [21], [22]. In most cases, hilly obstructions do not have single edges top. Rather more than one edges that are close together may be presented. In this case, the single rounded edge that will be fitted to vertex of the hill will have to enclose all the adjacent edges on the hilltop. In this case, the radius of curvature of the rounded edge may be well above what the value should have been if the rounded edge was to enclose only one edge on the hilltop. The study in this paper considers a situation where there are two adjacent edges on the hilltop to which a single rounded edge will be fitted and then used to determine the diffraction loss based on the ITU-R P.526-13 
method for rounded edge diffraction [20].

\section{The ITU-R P.526-13 Method for Diffraction over Single Rounded Edge}

The diffraction loss for single rounded obstacle according to Recommendation ITU-R P.526-13 is given as follows [20]:

$$
\mathrm{A}_{d B}=J(v)+\mathrm{T}(\mathrm{m}, \mathrm{n})
$$

where:

$J(v)$ is the Fresnel-Kirchhoff loss due to an equivalent knife-edge placed with its peak at the vertex point. According to ITU $-\mathrm{R} 526$, t he knife edge diffraction loss, J (v) is given as;

$$
\mathrm{J}(v)=6.9+20 \log \left[\left(\sqrt{(v-0.1)^{2}+1}\right)+v-0.1\right]
$$

$v$ is the diffraction parameter. The diffraction parameter $v$ is given as;

$$
\mathrm{v}=h \sqrt{\frac{2\left(d_{1}+d_{2}\right)}{\lambda\left(d_{1}\right)\left(d_{2}\right)}}
$$

where $K$ is the signal wavelength which is given as;

$$
\Lambda=\frac{c}{f}
$$

$\mathrm{f}$ is the frequency in $\mathrm{Hz}$ and $\mathrm{c}$ is the speed of light which is $3 \times 10^{8} \mathrm{~m} / \mathrm{s}$.

$\mathrm{h}$ is the line of sight (LOS) clearance which is obtained from the path profile and obstruction geometry, as shown in figure 2 .

$d_{1}$ is distance from the transmitter to the point where the LOS clearance is measured, as shown in figure 2.

$d_{2}$ is distance from the receiver to the point where the LOS clearance is measured, as shown in figure 2 .

$\mathrm{h}$ and $\lambda$ are in meters, and $\mathrm{d} 1$ and $\mathrm{d} 2$ are in kilometres.

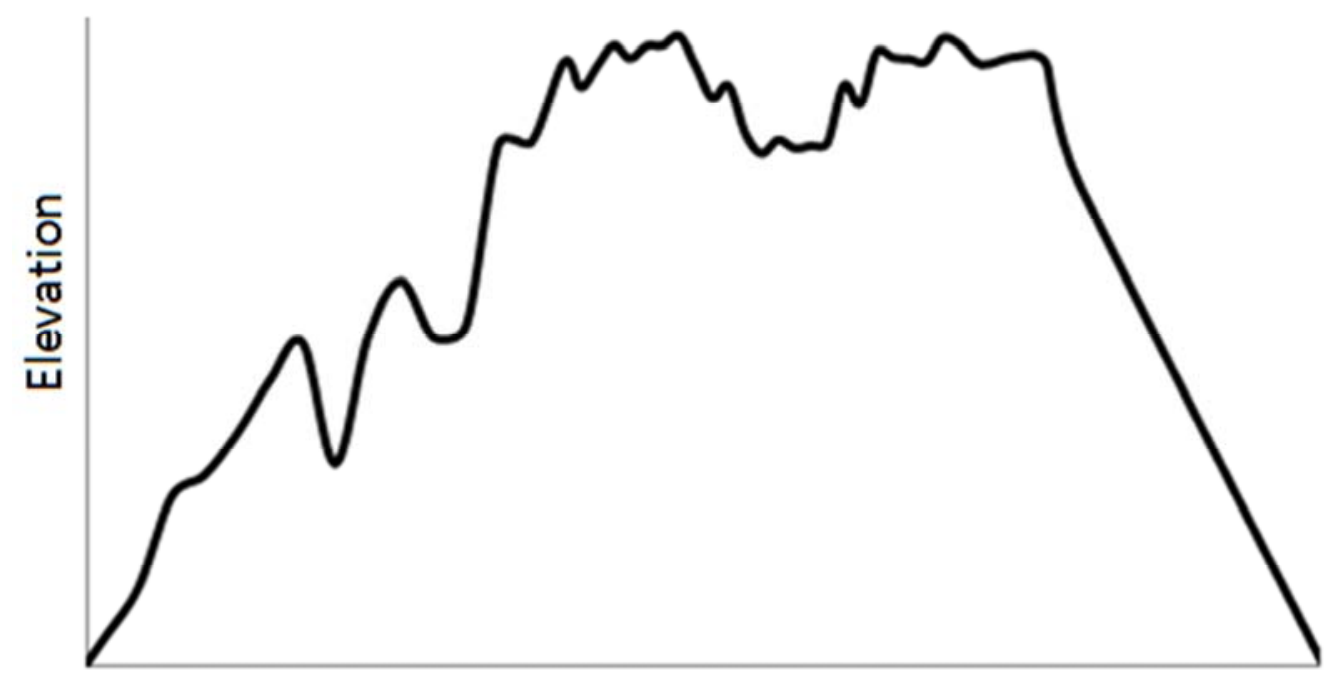

Figure 1. The path Profile Plot Of The Double Edged Hill.

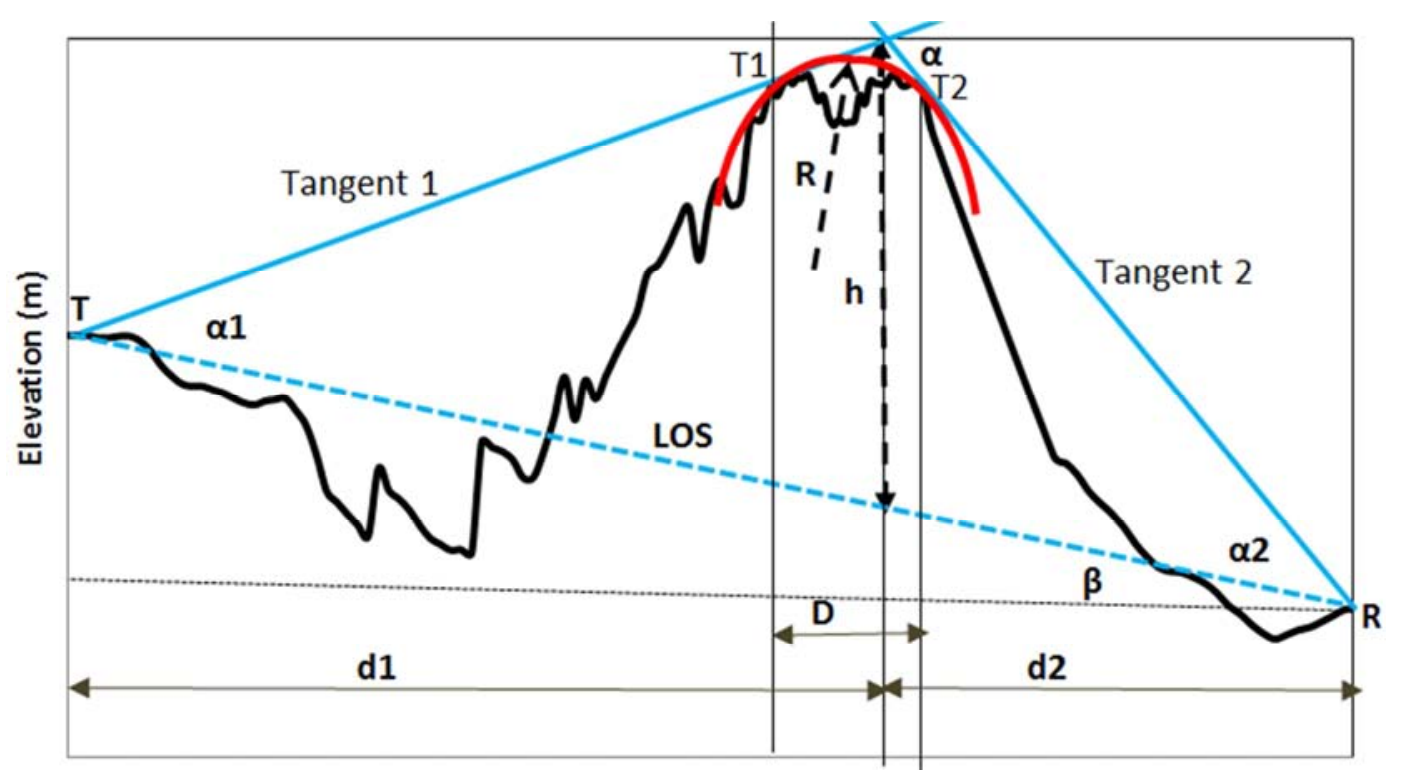

Figure 2. The Path Profile and Double Edged Hill Obstruction Geometry. 
$T(m, n)$ is the additional attenuation due to the curvature of the obstacle and it is given as [20]:

$$
\begin{gathered}
\mathrm{T}(\mathrm{m}, \mathrm{n}) \mathrm{dB}=7.2(m)^{1 / 2}-(2-12.5 n) m+3.6(m)^{3 / 2}- \\
0.8(m)^{2} \text { for } m n \leq 4 \\
\mathrm{~T}(\mathrm{~m}, \mathrm{n}) \mathrm{dB}=-6-20 \log (m n)+7.2(m)^{1 / 2}- \\
(2-17 n) m+3.6(m)^{3 / 2}-0.8(m)^{2} \text { for } m n>4
\end{gathered}
$$

Where,

$$
\begin{gathered}
\mathrm{m}=\frac{\mathrm{R}\left(\frac{(\mathrm{d} 1+\mathrm{d} 2)}{(\mathrm{d} 1)(\mathrm{d} 2)}\right)}{\left[\frac{\pi(R)}{\lambda}\right]^{1 / 3}} \\
n=\frac{h\left[\frac{\pi(R)}{\lambda}\right]^{2 / 3}}{R}
\end{gathered}
$$

The radius, $R$ of the circle fitted in the vicinity of the double edged hill vertex can be given the expression (Seybold, 2005, Barué, 200);

$$
R=\frac{2(\mathrm{D})\left(d_{1}\right)\left(d_{2}\right)}{(\alpha)\left[\left(d_{1}\right)^{2}+\left(d_{2}\right)^{2}\right]}
$$

where $\mathrm{D}$ is the occultation distance and it is obtained from the graph plot of the path profile and geometry of the obstruction, as shown in figure 2. Particularly, a line (referred here as tangent 1) is drawn from the transmitter to be tangential to the path profile at the vicinity of the hill apex. Let the tangent point of tangent 1 with the path profile be denoted as $\mathrm{T} 1$. Again, another line (referred here as tangent 2) is drawn from the receiver to be tangential to the path profile at the vicinity of the hill apex. Let the tangent point of tangent 1 with the path profile be denoted as T2. Then, D is the distance between T1 and T2. The point at which the tangent 1 and tangent 2 intersect above the hill vertex, as shown in figure 2 .

becomes the knife edge point. The LOS clearance is the height from the line of sight to the point of intersection of tangent 1and tangent 2 .

Let $\beta$ be the angle the LOS makes with the horizontal where;

$$
\beta=\tan ^{-1}\left(\frac{\mathrm{H}_{\mathbf{t}}-\mathrm{H}_{\mathbf{r}}}{\mathrm{d}}\right)
$$

where

$\mathrm{H}_{\mathbf{t}}$ is the height of the transmitter and $\mathrm{H}_{\mathbf{r}}$ is the height of the receiver and $\mathrm{d}$ is the distance between the transmitter and the receiver. The values of $d, H_{t}$ and $H_{r}$ are obtained from the path profile data.

$$
\mathrm{d}=\mathrm{d}_{1}+\mathrm{d}_{2}
$$

Let $\alpha_{1}$ be the angle (in radian) between the LOS and tangent 1 and let $\alpha_{2}$ be the angle (in radian) between the LOS and tangent 2 then, $\alpha$ is the external angle (in radian) between tangent 1 and tangent 2 at their point of intersection above the hill vertex, where

$$
\alpha=\alpha_{1}+\alpha_{2}
$$

The angles $\alpha_{1}$ and $\alpha_{2}$ are obtain by cosine rule as follows;

$$
\begin{gathered}
\operatorname{Cos}\left(\alpha_{1}\right)=\frac{\left(\mathrm{S}_{1}\right)^{2}+\left(\mathrm{S}_{3}\right)^{2}-\left(\mathrm{S}_{2}\right)^{2}}{2\left(\mathrm{~S}_{1}\right)\left(\mathrm{S}_{3}\right)} \\
\alpha_{1}=\operatorname{Cos}^{-1}\left(\frac{\left(\mathrm{S}_{1}\right)^{2}+\left(\mathrm{S}_{3}\right)^{2}-\left(\mathrm{S}_{2}\right)^{2}}{2\left(\mathrm{~S}_{1}\right)\left(\mathrm{S}_{3}\right)}\right)
\end{gathered}
$$

Similarly,

$$
\alpha_{2}=\operatorname{Cos}^{-1}\left(\frac{\left(\mathrm{S}_{2}\right)^{2}+\left(\mathrm{S}_{3}\right)^{2}-\left(\mathrm{S}_{1}\right)^{2}}{2\left(\mathrm{~S}_{2}\right)\left(\mathrm{S}_{3}\right)}\right)
$$

where

$\mathrm{S}_{1}$ is the length of the tangent 1 measured from the transmitter to the point of intersection of tangent 1 and tangent 2 , as shown in figure 2 .

$\mathrm{S}_{2}$ is the length of the tangent 2 measured from the receiver to the point of intersection of tangent 1 and tangent 2 , as shown in figure 2.

$\mathrm{S}_{3}$ is the length of the LOS measured from the transmitter to receiver

$\mathrm{S}_{1}, \mathrm{~S}_{2}$ and $\mathrm{S}_{3}$ are in meter and they are measured out from the

\begin{tabular}{|c|c|c|c|c|c|c|c|c|c|}
\hline Distance (m) & Elevation (m) & Distance (m) & Elevation (m) & Distance (m) & Elevation (m) & Distance (m) & Elevation (m) & Distance (m) & Elevation (m) \\
\hline 0.0 & 390.9 & 1206.9 & 379.8 & 2413.8 & 401.7 & 3180.8 & 411.8 & 4876.1 & 366.9 \\
\hline 40.2 & 390.9 & 1247.2 & 378.0 & 2454.1 & 397.3 & 3201.0 & 412.7 & 4921.4 & 367.5 \\
\hline 80.5 & 390.9 & 1287.4 & 377.2 & 2494.3 & 401.8 & 3221.2 & 412.5 & 4966.7 & 368.0 \\
\hline 120.7 & 390.7 & 1327.6 & 376.3 & 2534.5 & 403.9 & 3248.1 & 411.7 & 5011.9 & 368.0 \\
\hline 160.9 & 390.7 & 1367.8 & 375.0 & 2574.8 & 401.8 & 3288.3 & 412.0 & 5057.2 & 368.0 \\
\hline 201.2 & 390.9 & 1408.1 & 374.0 & 2615.0 & 402.3 & 3328.6 & 411.8 & 5102.4 & 368.0 \\
\hline 281.6 & 390.4 & 1488.5 & 372.9 & 2695.5 & 408.9 & 3835.2 & 380.8 & 5193.0 & 368.5 \\
\hline
\end{tabular}
path profile plot and the tangent line drawn on the path profile.

The line of sight (LOS) clearance, $h$ is given as;

$$
h=\frac{S_{1}\left[\operatorname{Sin}\left(\alpha_{1}\right)\right]}{\operatorname{Sin}(90-\beta)}
$$

\section{Results and Discussions}

The study if conducted for the L-band microwave frequency which ranges from $1 \mathrm{GHz}$ to $2 \mathrm{GHz}$. Specifically, the $1 \mathrm{GHz}$ and $1.9 \mathrm{GHz}$ frequencies are considered in this paper. The elevation profile data used for the study is given in Table 1. From Table 1 the maximum elevation is $412.75 \mathrm{~m}$ and it occurred at a distance of $2877.3 \mathrm{~m}$ from the transmitter.

Table 1. The Elevation Profile For The Plateau. 


\begin{tabular}{|c|c|c|c|c|c|c|c|c|c|}
\hline Distance (m) & Elevation (m) & Distance (m) & Elevation (m) & Distance (m) & Elevation (m) & Distance (m) & Elevation (m) & Distance (m) & Elevation (m) \\
\hline 321.8 & 389.6 & 1528.8 & 373.0 & 2735.7 & 411.8 & 3880.4 & 380.3 & 5238.2 & 369.0 \\
\hline 362.1 & 388.4 & 1569.0 & 372.7 & 2755.9 & 410.9 & 3925.7 & 379.3 & 5283.5 & 369.3 \\
\hline 402.3 & 387.5 & 1609.2 & 382.0 & 2776.1 & 411.7 & 3971.0 & 377.9 & 5328.7 & 369.4 \\
\hline 442.5 & 386.9 & 1649.5 & 381.5 & 2796.4 & 412.4 & 4016.2 & 376.9 & 5374.0 & 368.9 \\
\hline 482.8 & 386.7 & 1689.7 & 381.2 & 2816.6 & 411.9 & 4061.5 & 376.0 & 5419.3 & 368.3 \\
\hline 523.0 & 386.7 & 1729.9 & 380.5 & 2836.8 & 412.4 & 4106.7 & 374.9 & 5464.5 & 368.0 \\
\hline 603.5 & 386.1 & 1810.4 & 379.1 & 2877.3 & 412.8 & 4197.2 & 372.4 & 5555.0 & 368.7 \\
\hline 643.7 & 385.6 & 1850.6 & 381.3 & 2897.5 & 411.7 & 4242.5 & 371.5 & 5600.3 & 367.5 \\
\hline 683.9 & 385.2 & 1890.8 & 384.0 & 2917.8 & 410.5 & 4287.8 & 371.2 & 5645.6 & 366.9 \\
\hline 724.2 & 385.1 & 1931.1 & 387.5 & 2938.0 & 411.0 & 4333.0 & 371.1 & 5690.8 & 366.3 \\
\hline 764.4 & 385.4 & 1971.3 & 383.8 & 2958.2 & 409.2 & 4378.3 & 370.7 & 5736.1 & 366.4 \\
\hline 804.6 & 385.5 & 2011.5 & 387.2 & 2978.5 & 408.5 & 4423.5 & 370.2 & 5781.3 & 366.4 \\
\hline 844.8 & 385.6 & 2051.8 & 385.6 & 2998.7 & 409.0 & 4468.8 & 369.4 & 5826.6 & 366.2 \\
\hline 925.3 & 383.4 & 2132.2 & 389.4 & 3039.1 & 408.8 & 4559.3 & 367.6 & 5917.1 & 366.0 \\
\hline 965.5 & 380.9 & 2172.5 & 391.1 & 3059.4 & 408.9 & 4604.6 & 366.7 & 5962.4 & 366.9 \\
\hline 1005.8 & 377.9 & 2212.7 & 392.8 & 3079.6 & 411.0 & 4649.8 & 366.0 & 6007.6 & 367.7 \\
\hline 1046.0 & 377.0 & 2252.9 & 396.1 & 3099.8 & 410.3 & 4695.1 & 365.5 & 6052.9 & 368.6 \\
\hline 1086.2 & 376.0 & 2293.1 & 396.8 & 3120.1 & 412.2 & 4740.4 & 365.9 & 6098.1 & 370.9 \\
\hline 1126.5 & 375.0 & 2333.4 & 398.3 & 3140.3 & 412.0 & 4785.6 & 366.3 & 6143.4 & 370.9 \\
\hline 1166.7 & 374.1 & 2373.6 & 400.3 & 3160.5 & 411.9 & 4830.9 & 366.5 & 6188.7 & 370.9 \\
\hline
\end{tabular}

Table 2. The diffraction loss and associated parameters for the double edged hilltop using the Recommendation ITU-R P.526-13 model.

\begin{tabular}{|c|c|c|}
\hline f (GHz) & Frequency & 6000 \\
\hline$\lambda(\mathrm{m})$ & Wavelength & 0.05 \\
\hline $\mathrm{S} 1(\mathrm{~m})$ & The length of the tangent from the transmitter to the intersection point of the two tangent & 3086.7642 \\
\hline $\mathrm{S} 2(\mathrm{~m})$ & the length of the tangent from the receiver to the intersection point of the two tangents & 3102.3379 \\
\hline $\mathrm{S} 3(\mathrm{~m})$ & the length of the tangent from the receiver from the transmitter & 6188.6974 \\
\hline $\mathrm{d} 1(\mathrm{~m})$ & the distance from the transmitter to the intersection point of the two tangents, that is point & 3086.6596 \\
\hline $\mathrm{d} 2(\mathrm{~m})$ & the distance from the receiver to the intersection point of the two tangents & 3102.0054 \\
\hline $\mathrm{d}(\mathrm{m})$ & the distance from the transmitter to the receiver & 6188.665 \\
\hline$\alpha \mathrm{t}$ (radian) & The angle the tangent line from the transmitter makes with the LOS & 0.0114088 \\
\hline$\alpha \mathrm{r}$ (radian) & The angle the tangent line from the receiver makes with the LOS & 0.0114664 \\
\hline$\alpha$ (radian) & Sum of angles $\alpha \mathrm{t}$ and $\alpha \mathrm{r}$ & 0.0228753 \\
\hline$\beta$ (radian) & The angle the LOS makes with the horizontal & 0.0032335 \\
\hline $\mathrm{h}(\mathrm{m})$ & The LOS clearance height & 35.393521 \\
\hline $\mathrm{D}(\mathrm{m})$ & The occultation distance & 532.203 \\
\hline $\mathrm{n}$ & & 19.596466 \\
\hline $\mathrm{k}$ & & 243.35759 \\
\hline $\mathrm{b}$ & & 1 \\
\hline $\mathrm{V}$ & Diffraction parameter for the ITU-R P.526-13 Method & 0.1798348 \\
\hline $\mathrm{R}(\mathrm{m})$ & The radius of the circle fitted in the vicinity of the hill vertex & 23265.392 \\
\hline $\mathrm{J}(\mathrm{v})$ & & 7.5927016 \\
\hline $\mathrm{T}(\mathrm{m}, \mathrm{n})$ & & 32.243248 \\
\hline $\mathrm{A}(\mathrm{dB})$ & The diffraction loss for the double edged hilltop using the Recommendation ITU-R P.526-13 model & 42.563065 \\
\hline
\end{tabular}

Table 2 shows diffraction loss and associated parameters for the double edged hilltop using the Recommendation ITU-R P.526-13 model. From Table 2, the path length (d) is $6188.665 \mathrm{~m}$. Also, the tangent from the transmitter and the tangent from the receiver intersected at a distance of $3086.6596 \mathrm{~m}$ from the transmitter and a distance of $3102.0054 \mathrm{~m}$ from the receiver. The line of sight makes an angle of 0.0032335 radians with the horizontal. The LOS clearance height is $35.393521 \mathrm{~m}$. The occultation distance is $532.203 \mathrm{~m}$. The diffraction loss computed for the double edged hilltop using the Recommendation ITU-R P.526-13 model is $42.563065 \mathrm{~dB}$.

\section{Conclusions}

Recommendation ITU-R P.526-13 model for rounded edge diffraction loss method is presented. The method is used to determine the diffraction loss over a double edged hilltop in the path of $6 \mathrm{GHz} \mathrm{C}$-band microwave signal. The computation is based on the path profile of a cases study with double edged hilltop. 


\section{References}

[1] Mahafza, B. R. (2016). Radar signal analysis and processing using MATLAB. CRC Press.

[2] Bai, T., \& Heath, R. W. (2015). Coverage and rate analysis for millimeter-wave cellular networks. IEEE Transactions on Wireless Communications, 14 (2), 1100-1114.

[3] Pyattaev, A., Johnsson, K., Andreev, S., \& Koucheryavy, Y. (2015). Communication challenges in high-density deployments of wearable wireless devices. IEEE Wireless Communications, 22 (1), 12-18.

[4] Sachdeva, N., \& Sharma, D. (2012). Diversity: A fading reduction technique. International Journal of Advanced Research in Computer Science and Software Engineering, ISSN.

[5] Patwari, N. ECE 5325/6325: Wireless Communication Systems Lecture Notes, Spring 2010.

[6] Gunnarsson, F., Amirijoo, M., \& Moe, J. (2016). U. S. Patent No. 9,264,919. Washington, DC: U. S. Patent and Trademark Office.

[7] Pradhan, C., \& Murthy, G. R. (2015). Analysis of Path Loss mitigation through Dynamic Spectrum Access: Software Defined Radio. arXiv preprint arXiv: 1510.04772.

[8] Sai Sanath Kumar, K., Reddy, K. N. K., Pushpavathy, V., Reddy, P. R., Sharma, D., Sharma, P. K., \& Krishna, B. V. (2016). Calculation of Path Losses at CM3 for Wireless Body Area Networks (WBAN) by using Different Types of Antennas. International Journal of Applied Engineering Research, 11 (7), 5210-5217.

[9] Agrawal, S., Gupta, N., \& Shrivastava, M. (2016). Impact of GSM Spectrum Auction in $900 \& 1800 \mathrm{MHz}$ Band. International Transactions on Electronics and Communication Engineering, 1 (1), 18-27.

[10] Malila, B., Falowo, O., \& Ventura, N. (2015, September). Millimeter wave small cell backhaul: An analysis of diffraction loss in NLOS links in urban canyons. In AFRICON, 2015 (pp. 1-5). IEEE.

[11] Saman, M. A., Ismail, A. F., Badron, K., Ramli, H. A. M.,
Hashim, W., \& Fakrullah, A. N. A. (2015). Development of Spectrum Management Tool for Malaysia Using Open-Source GIS Software. In Theory and Applications of Applied Electromagnetics (pp. 127-135). Springer International Publishing.

[12] Vargas, T. A. A., Peña, J. E. A., Medina, J. G. B., Pulido, J. D. Z., \& Peñaloza, M. L. S. (2015, October). Configuration of propagation models for computational simulation of different telecommunications services. In Engineering Applications-International Congress on Engineering (WEA), 2015 Workshop on (pp. 1-6). IEEE.

[13] Jude, O. O., Jimoh, A. J., \& Eunice, A. B. (2016). Software for Fresnel-Kirchoff Single Knife-Edge Diffraction Loss Model. Mathematical and Software Engineering, 2 (2), 76-84.

[14] Adebayo, T. L., \& Edeko, F. O. (2007). Investigation and Modeling of Diffraction Loss at $1.8 \mathrm{GHZ}$ in a Mountainous Terrain: A Case Study of Olumo Rock, Abeokuta, Nigeria. Research Journal of Applied Sciences, 2 (1), 60-64.

[15] Baldassaro, P. M. (2001). RF and GIS: Field Strength Prediction for Frequencies between $900 \mathrm{MHz}$ and $28 \mathrm{GHz}$.

[16] Parsons, J. D., \& Parsons, P. J. D. (2000). The mobile radio propagation channel. Second edition. John Wiley \& Sons.

[17] Hacking, K. U. H. F. (1968). Propagation over rounded hills. BBC Research Department. Research Report No. RA-21, 30.

[18] Rice, P. L., Longley, A. G., Norton, K. A., \& Barsis, A. P. (1967). Transmission Loss Predictions For Tropospheric Communication Circuits, Volume 1. Institute For Telecommunication Sciences And Aeronomy Boulder Co

[19] Wait JR Conda AM (1959) Diffraction of electromagnetic waves by smooth obstacles for grazing angles. Journal of Research of the N. B. S. vol 63D

[20] ITU-R P.526-13 (11/2013) Propagation by Diffraction. Available online http://www.itu.int/rec/RREC-P.526-13-201311-I

[21] Barué, G. (2008). Microwave engineering: land \& space radiocommunications (Vol. 9). John Wiley \& Sons.

[22] De Assis, M. (1971). A simplified solution to the problem of multiple diffraction over rounded obstacles. IEEE Transactions on Antennas and Propagation, 19 (2), 292-295. 